\title{
Clinical Effect of Shenfu Injection in Patients with Septic Shock: A Meta-Analysis and Systematic Review
}

\author{
Zijun Mou, ${ }^{1}$ Zhengtao Lv, ${ }^{2}$ Yi Li, ${ }^{1}$ Meng Wang, ${ }^{3}$ Qun Xu, ${ }^{3}$ and Xuezhong Yu \\ ${ }^{1}$ Emergency Department, Peking Union Medical College Hospital, Beijing 100730, China \\ ${ }^{2}$ Department of Orthopedics, Tongji Hospital, Tongji Medical College, Huazhong University of Science and Technology, \\ Wuhan 430030, China \\ ${ }^{3}$ Department of Epidemiology and Biostatistics, Institute of Basic Medical Sciences Chinese Academy of Medical Sciences, \\ School of Basic Medicine Peking Union Medical College, Beijing 100005, China \\ Correspondence should be addressed to Yi Li; billliyi@yahoo.com and Xuezhong Yu; erxuezhongyu@sina.cn
}

Received 24 February 2015; Revised 15 May 2015; Accepted 18 May 2015

Academic Editor: Christian Lehmann

Copyright (c) 2015 Zijun Mou et al. This is an open access article distributed under the Creative Commons Attribution License, which permits unrestricted use, distribution, and reproduction in any medium, provided the original work is properly cited.

Purpose. To conduct a meta-analysis evaluating the efficacy of Shenfu injection for treating patients with septic shock when compared with conventional therapy. Methods. Eight databases including Pubmed, EMBASE, Cochrane Library, ISI Web of Science, CNKI, Wanfang, VIP, and CBM were searched up to October 2014. Randomized controlled trials assessing the efficacy of Shenfu injection were identified. Mean arterial pressure, heart rate, lactate, and mortality were included as outcome measurements. Results. We analyzed data from 12 randomized controlled trials involving 904 participants. Compared with conventional therapy, Shenfu injection could further increase the mean arterial pressure at 1 hour (SMD 0.38; 95\% CI, 0.01-0.74) and 6 hours (SMD 0.82; 95\% CI, 0.03-1.61). Shenfu injection could further normalize heart rate at 6 hours (SMD $-0.90 ; 95 \%$ CI, $-1.47-0.33$ ) and clear serum lactate at 6 hours (SMD $-0.51 ; 95 \% \mathrm{CI},-0.70-0.32$ ) and 24 hours (SMD, 0.52; 95\% CI, -0.77-0.26). As the endpoint of mortality was not unified, it was not meta-analyzed. Conclusions. Based on the findings in present review, Shenfu injection is more effective than conventional therapy in increasing mean arterial pressure, normalizing heart rate, clearing serum lactate, and reducing mortality. These results should be confirmed in higher level clinical trials in the future.

\section{Introduction}

Septic shock is characterized by an alteration in tissue perfusion associated with persistent arterial hypotension [1]. It is generally defined as systolic arterial pressure of less than $90 \mathrm{~mm} \mathrm{Hg}$, despite adequate fluid resuscitation [2]. This leads to organ dysfunction and even death in around $50 \%$ of cases [3].

The fundamental principles for the management of sepsis include early recognition, control of the infection source, appropriate and timely administration of antimicrobial drugs, and resuscitation with intravenous fluids and vasoactive drugs [4]. Fluid resuscitation is essential for the restoration and maintenance of adequate intravascular volume and organ perfusion [5]. The goal of cardiovascular resuscitation of septic shock is to improve organ perfusion, often by increasing the mean arterial pressure (MAP) [6].
Recommendations suggest that MAP of around $65 \mathrm{~mm} \mathrm{Hg}$ should initially be targeted, since MAP below this value may be associated with a worse evolution $[7,8]$.

Once fluid resuscitation is insufficient to restore hemodynamic stability, vasopressor therapy is typically required to restore organ perfusion. Although norepinephrine is the current recommended mainstay of sepsis treatment for hypotension, excessive adrenergic stress has multiple adverse effects including direct myocardial damage, insulin resistance, thrombogenicity, immunosuppression, and enhanced bacterial growth [9]. High plasma catecholamine levels, the extent and duration of catecholamine therapy, and tachycardia are all independently associated with poor outcomes in critically ill patients $[10,11]$.

Septic patients often have an elevated heart rate (HR), even after excluding common causes of tachycardia such as hypovolemia, anemia, pain, and agitation [11]. An elevated 
HR is associated with adverse outcomes in septic shock and thus represents the extent of disease severity [12]. Heart rate reduction considerably lowers cardiac energy demands, thereby creating a better balance between myocardial energy generation and expenditure in conditions of impaired energy production [13]. In addition, sepsis impairs the ability of tissues to extract oxygen so that adenosine triphosphate (ATP) generation from glucose oxidation is supplemented by ATP generation from glycolysis, leading to lactate production [14]. Presence of elevated lactate levels was associated with a significantly increased mortality in patients with vasopressordependent septic shock and this hyperlactatemia represents a persistent perfusion deficit [15]. Thus, elevated blood lactate should be a trigger for early escalation of care, including resuscitation, irrespective of arterial blood pressure [16, 17].

Shenfu injection is a well-known Chinese traditional medicine, which is made of Red Radix Ginseng and Radix Aconitum carmichaelii, with the active ingredients of ginseng saponin and aconitum alkaloids. Its clinical indications include Tuojue in the Chinese traditional medicine (meaning shock). Pharmacological studies have shown that it could elevate blood pressure and improve microcirculation against inflammatory reaction.

Shenfu injection as a common emergency medicine is frequently used in the national hospital emergency department, showing good curative effect in rescuing septic shock and resuscitation $[18,19]$. However, there is little published information to warrant Shenfu injection as a standard treatment of septic shock. The aim of our current study is to evaluate the clinical effect of Shenfu injection when compared with conventional therapy based on randomized controlled trials (RCTs).

\section{Material and Methods}

2.1. Literature Search Strategy. A systematic literature search of Cochrane Library, EMBASE, ISI Web of Science, and PubMed was conducted. Chinese databases including China Knowledge Resource Integrated (CNKI) Database, Wanfang Database, VIP, and Chinese Biomedical Literature (CBM) Database were also scrutinized for the identification of trials. All the above databases were searched from their inception dates up to the latest issue (October 2014). No language restriction was used.

The following medical subject headings or keywords were used for English databases: Shenfu, septic shock, and toxic shock. For Chinese databases we used free text terms "shenfu" and "nong du xing xiu ke" or "gan ran xing xiu ke." In addition, the bibliographies of relevant systematic reviews and clinical guidelines were manually searched. The reference section of each study was also searched.

2.2. Inclusion Criteria. Studies that met the following criteria were included: (a) the enrolled patients were diagnosed with septic shock, the age of enrolled patients was $>16$ years, no restrictions on race or sex were imposed, and the number of included patients in both groups had to be $>10$; (b) the included studies were required to be randomized controlled trials aiming to assess the efficacy of Shenfu injection for septic shock; (c) experimental groups mainly received Shenfu injection and conventional therapy, without differentiating the administration method of Shenfu injection; (d) patients in control groups were treated in conventional ways, which included anti-infection, fluid resuscitation, nutrition support, correct acidosis, and administration of dopamine, and the drugs administered in experimental groups had to be in accordance with the drugs utilized in control groups; (e) the outcome measurements had to include the MAP, HR, lactate, or mortality.

2.3. Exclusion Criteria. The three exclusion criteria for each identified study were as follows: (a) articles regarding animal experiments, review articles, case reports, or expert experience reports; (b) nonrandomized studies; (c) studies that were duplicates for retrieving or publishing.

2.4. Data Extraction. Two investigators (Zhengtao Lv and Zijun Mou) screened each article independently and each one was blinded to the findings of the other reviewer. In accordance with the predetermined inclusion and exclusion criteria, two reviewers independently performed a strict screening to identify qualified articles, and they extracted data independently from these eligible articles using a standardized collection form, which included first author, year of publication, study design, cohort sizes, baseline characteristics for participants in different groups, guidelines for management, intervention treatments, main outcome assessments, timing of outcome measure, and follow-up periods after treatments. If the required information was not available in the included studies, attempts were made to contact the authors of the original papers via e-mail. Any disagreement between reviewers was resolved through discussion until a consensus was reached. The third review author (Yi Li) was consulted in case a consensus could not be reached.

2.5. Quality Assessment. The Cochrane Collaboration's tool was used to assess the risk of bias in included studies, which was based on seven items: random sequence generation, allocation concealment, blinding of participants and personnel, blinding of outcome assessment, incomplete outcome data, selective reporting, and other sources of bias [20]. The response for each criterion was reported as low risk of bias, high risk of bias, and unclear risk of bias. Two reviewers evaluated the quality of trials independently.

2.6. Data Synthesis and Analysis. The meta-analysis and statistical analyses were performed by using RevMan 5.3 analyses software of the Cochrane Collaboration. Odds ratio (OR) and the associated 95\% confidence intervals (CIs) were calculated for mortality. The standard mean difference (SMD) was calculated for MAP, HR, and serum lactate using the same methodology. Before the data of included studies was combined, heterogeneity between trial results was estimated using a standard chi-square test and the Higgins $I^{2}$ test ( $P>0.1$ and $I^{2}<50 \%$ indicate acceptable heterogeneity). We pooled data across studies using random effect models 


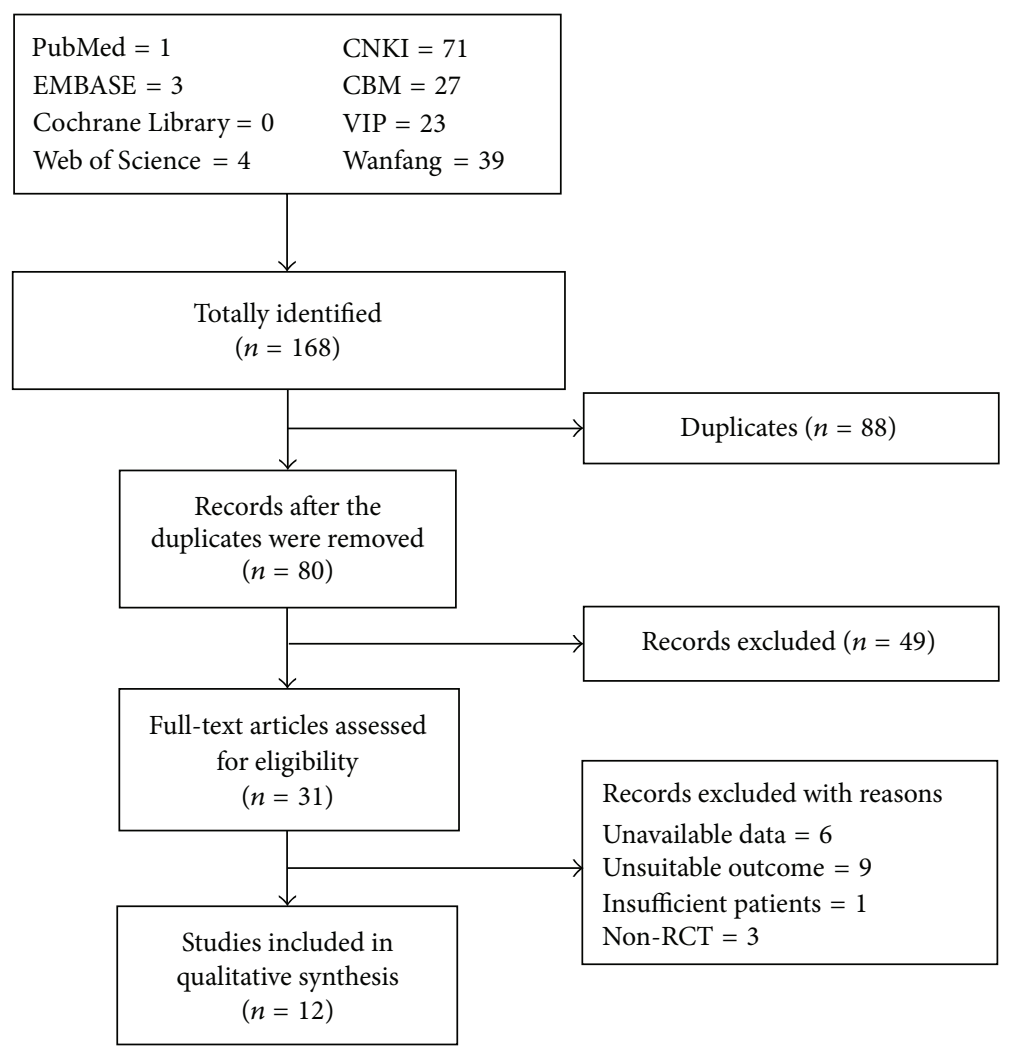

FIGURE 1: Flowchart of the literature search and study selection.

if statistical heterogeneity existed; otherwise, a fixed effect model would be used. In case of heterogeneity, subgroup analysis was conducted. Publication bias was assessed via a funnel plot if the number of included studies is equal to or greater than 5 .

\section{Results}

3.1. Literature Search Results. An initial search of RCTs yielded 168 potential literature citations, including 39 records from Wanfang, 71 from CNKI, 23 from VIP, 27 from CBM, 1 from PubMed, 0 from Cochrane Library, 3 from EMBASE, and 4 from ISI Web of Science. 88 studies were deleted because they were duplicates. According to the predetermined selection criteria, 31 potentially relevant studies were selected and retrieved for a full-text assessment after screening titles and abstracts. Of the remaining 31 articles, 6 studies were excluded because their data was unavailable, 9 studies were excluded because they employed unsuitable outcome, 1 study was excluded because the number of included patients was $<10$, and 3 studies were excluded because they were not RCTs. Finally, 12 studies met our inclusion criteria and were included in the meta-analysis. The literature screening process is summarized in a flowchart (Figure 1).

3.2. Study Characteristics. The main characteristics of the 12 trials are listed in Tables 1 and 2. These studies were all conducted by Chinese investigators and published between
2007 and 2014. Each study was performed at a single center. The 12 RCTs included a total of 904 patients with septic shock: 464 patients in the Shenfu injection group and 440 patients in the control group. Age of the participants ranged from 16 to 83 years. All 12 trials used 2-parallelarm designs; Shenfu injection plus conventional therapy was compared with conventional therapy in these RCTs. Four studies followed the "surviving sepsis campaign guidelines for management of severe sepsis and septic shock" published in 2004 (SSC 2004) [21]; three studies followed the "surviving sepsis campaign: international guidelines for management of severe sepsis and septic shock" published in 2008 (SSC 2008) [7]; the guidelines followed by the other five studies were not reported. The timing of outcome measurements ranged from 1 hour to 28 days. Only one study [19] mentioned follow-up after treatment. Seven studies [18, 22-27] employed MAP as outcome measure, eight studies [18, 22-28] employed HR as outcome measure, eight studies $[18,22,23,25,26,29,30]$ utilized lactate as outcome measure, and seven studies [18, 19, $23,25,27,30,31]$ employed mortality as outcome measure.

3.3. Risk of Bias. The methodological quality of selected trials was assessed using the Cochrane Collaboration's tool. All of the studies included suggested randomization, but only five studies reported the method of random sequences generation. All studies failed to report details about allocation concealment. The blinding of outcome measurement was judged to low risk of bias because the outcomes were unlikely 
TABLE 1: Characteristics of included trials.

\begin{tabular}{|c|c|c|c|c|}
\hline Authors, year & Nation & Study design & Sample size $(n 1 / n 2)$ & $\begin{array}{l}\text { Age (mean or } \\
\text { range) }\end{array}$ \\
\hline Sun et al., 2007 [24] & China & RCT & $60(34 / 26)$ & $\begin{array}{l}\text { E: } 18-65 ; \\
C: 18-60\end{array}$ \\
\hline Sun et al., 2008 [31] & China & RCT & $60(30 / 30)$ & $\begin{array}{l}\text { E: } 18-65 ; \\
\text { C: } 18-60\end{array}$ \\
\hline Zhang et al., 2011 [27] & China & RCT & $93(48 / 45)$ & $\begin{array}{l}\text { E: } 16-72 ; \\
\text { C: } 17-70\end{array}$ \\
\hline Li et al., 2013 [29] & China & RCT & $60(30 / 30)$ & $\begin{array}{l}\text { E: } 25-82 ; \\
\text { C: } 22-79\end{array}$ \\
\hline Lin et al., 2012 [28] & China & RCT & $120(60 / 60)$ & $\begin{array}{l}\text { E: } 32-81 ; \\
\text { C: } 31-83\end{array}$ \\
\hline $\mathrm{Xu}$ and $\mathrm{Xu}, 2012$ [25] & China & RCT & $49(22 / 27)$ & $\begin{array}{l}\text { E: } 54 \pm 0.3 ; \\
\text { C: } 55 \pm 0.2\end{array}$ \\
\hline Xu et al., 2013 [26] & China & RCT & $98(48 / 50)$ & $\begin{array}{l}\text { E: } 57 \pm 7.0 \\
\text { C: } 60 \pm 8.0\end{array}$ \\
\hline He et al., 2013 [19] & China & RCT & $64(32 / 32)$ & Not reported \\
\hline Lin et al., 2013 [30] & China & RCT & $49(33 / 16)$ & $\begin{array}{l}\text { E: } 79.8 \pm 12.6 \\
\text { C: } 76.5 \pm 13.2\end{array}$ \\
\hline Lv et al., 2013 [23] & China & RCT & $52(27 / 25)$ & Not reported \\
\hline Dong and Shen, 2014 [18] & China & RCT & $91(46 / 45)$ & $\begin{array}{l}\text { E: } 68.34 ; \\
\text { C: } 69.56\end{array}$ \\
\hline Liu et al., 2014 [22] & China & RCT & $108(54 / 54)$ & Not reported \\
\hline
\end{tabular}

to be influenced by lack of blinding but there was high risk bias for blinding the participants or personnel in all studies. The number of dropouts and reasons for withdrawal were not reported in any of the above studies. When it comes to selective reporting bias, there was a low risk of bias since we only included the studies which used HR, MAP, lactate, or mortality as outcome measures. All studies had low risk of other biases except two studies that did not report baseline similarity. Finally, all studies were judged to be of a poor methodological quality. The judgment of risk of bias was presented in corresponding forest plots (Figures 2, 3, and 4).

\subsection{Meta-Analyses Results}

3.4.1. MAP. Seven studies [18, 22-27] employed MAP as outcome measure; since there was obvious heterogeneity among included studies $\left(\tau^{2}=0.59 ; \chi^{2}=89.94\right.$, degree of freedom $\left.(\mathrm{df})=8(P<0.00001) ; I^{2}=91 \%\right)$, the random effect model was utilized for statistical analysis. The pooled MAP at 1 hour after treatment indicated that Shenfu injection further increased MAP when compared with conventional care $(0.38[0.01,0.74])$; the pooled MAP at 6 hours after treatment indicated that Shenfu injection further increased MAP when compared with conventional care $(0.82[0.03,1.61])$ (Figure 2).

3.4.2. HR. Eight studies [18, 22-28] employed HR as outcome measure; since there was obvious heterogeneity among included studies $\left(\tau^{2}=0.60 ; \chi^{2}=131.18\right.$, df $=11(P<$ $\left.0.00001) ; I^{2}=92 \%\right)$, the random effect model was utilized for statistical analysis. The pooled HR at 1 hour after treatment indicated that there was no significant difference between Shenfu injection and conventional care $(-0.21[-0.89,0.47])$, the pooled $\mathrm{HR}$ at 6 hours after treatment indicated that Shenfu injection further reduced HR when compared with conventional care $(-0.90[-1.47,-0.33])$, and the pooled HR at 24 hours after treatment indicated that there was no significant difference between Shenfu injection and conventional care $(-0.04[-1.24,1.16])$ (Figure 3$)$.

3.4.3. Lactate. Eight studies $[18,22,23,25,26,29,30]$ utilized lactate as outcome measure. Fixed effect model was used for statistical analysis because there was no obvious heterogeneity among studies. The pooled lactate at 1 hour after treatment indicated that there was no significant difference between Shenfu injection and conventional care $(-0.15[-0.43,0.12])$, the pooled lactate at 6 hours after treatment indicated that Shenfu injection further cleared serum lactate when compared with conventional care $(-0.51$ $[-0.70,-0.32])$, and the pooled lactate at 24 hours after treatment indicated that Shenfu injection further reduced serum lactate when compared with conventional care $(-0.52$ $[-0.77,-0.26])$ (Figure 4).

3.4.4. Mortality. Seven studies [18, 19, 23, 25, 27, 30, 31] employed mortality as outcome. As the endpoint of outcome measure was not unified, we only listed the mortality rate and the corresponding endpoint in Table 3. Except in Dong and Shen's study, a decreased trend of mortality and statistical difference could be detected in the other six studies. 


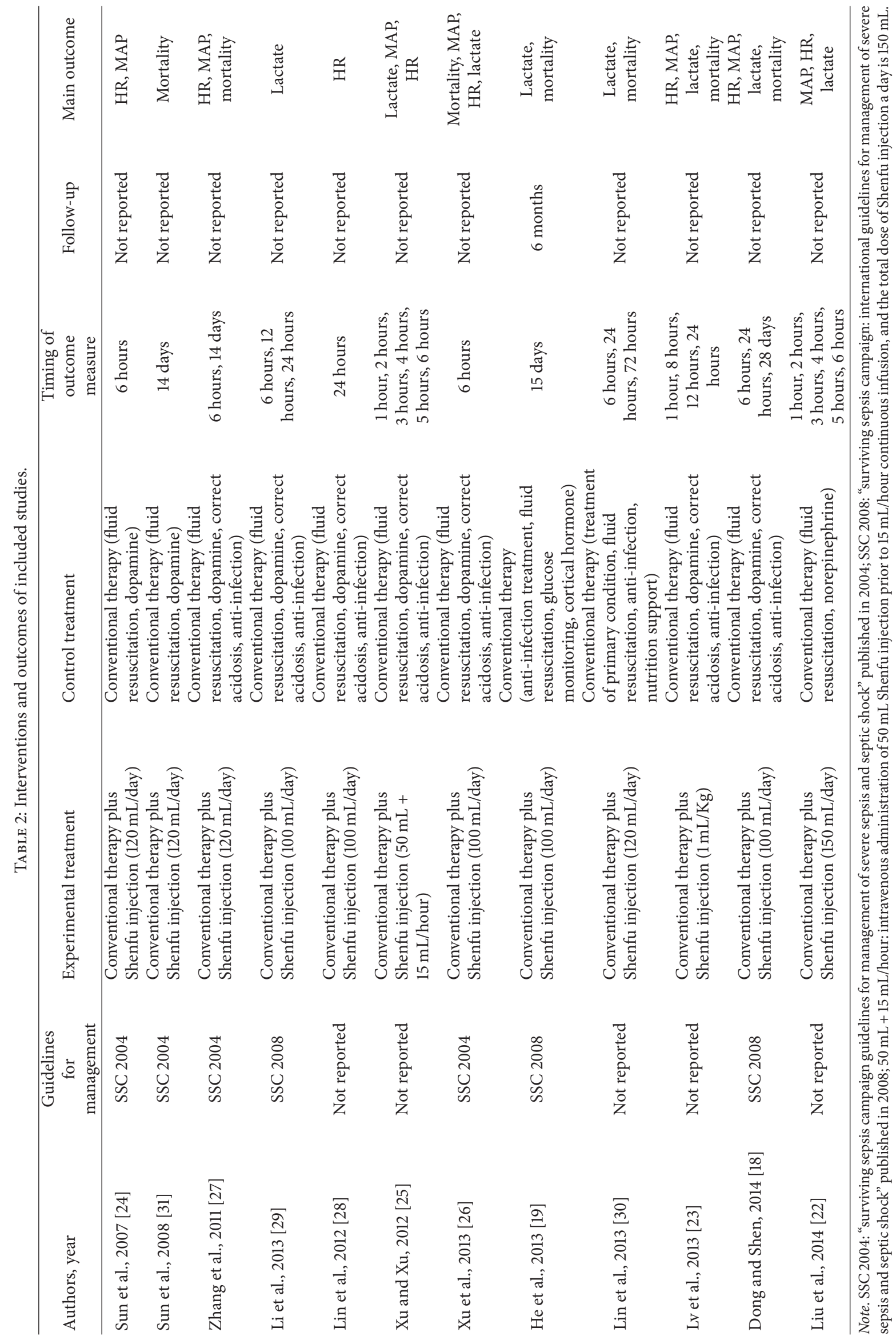


TABle 3: Mortality of experimental and control groups and the associated $P$ value.

\begin{tabular}{|c|c|c|c|c|}
\hline Authors, year & Endpoint & Experimental group & Control group & $P$ value \\
\hline Sun et al., 2008 [31] & 14 days & $6.67 \%(2 / 30)$ & $13.33 \%(4 / 30)$ & $P<0.05$ \\
\hline Zhang et al., 2011 [27] & 14 days & $4.17 \%(2 / 48)$ & $15.56 \%(7 / 45)$ & $P<0.05$ \\
\hline Xu et al., 2013 [26] & 72 hours & $12.50 \%(6 / 48)$ & $26.00 \%(13 / 50)$ & $P<0.05$ \\
\hline He et al., 2013 [19] & 28 days & $3.13 \%(1 / 32)$ & $9.38 \%(3 / 32)$ & $P<0.01$ \\
\hline Lin et al., 2013 [30] & 24 hours & $9.09 \%(3 / 33)$ & $37.50 \%(6 / 16)$ & $P<0.05$ \\
\hline Lv et al., 2013 [23] & 24 hours & $7.40 \%(2 / 27)$ & $24.00 \%(6 / 25)$ & $P<0.05$ \\
\hline Dong and Shen, 2014 [18] & 28 days & $56.52 \%(26 / 46)$ & $64.44 \%(29 / 45)$ & $P>0.05$ \\
\hline
\end{tabular}

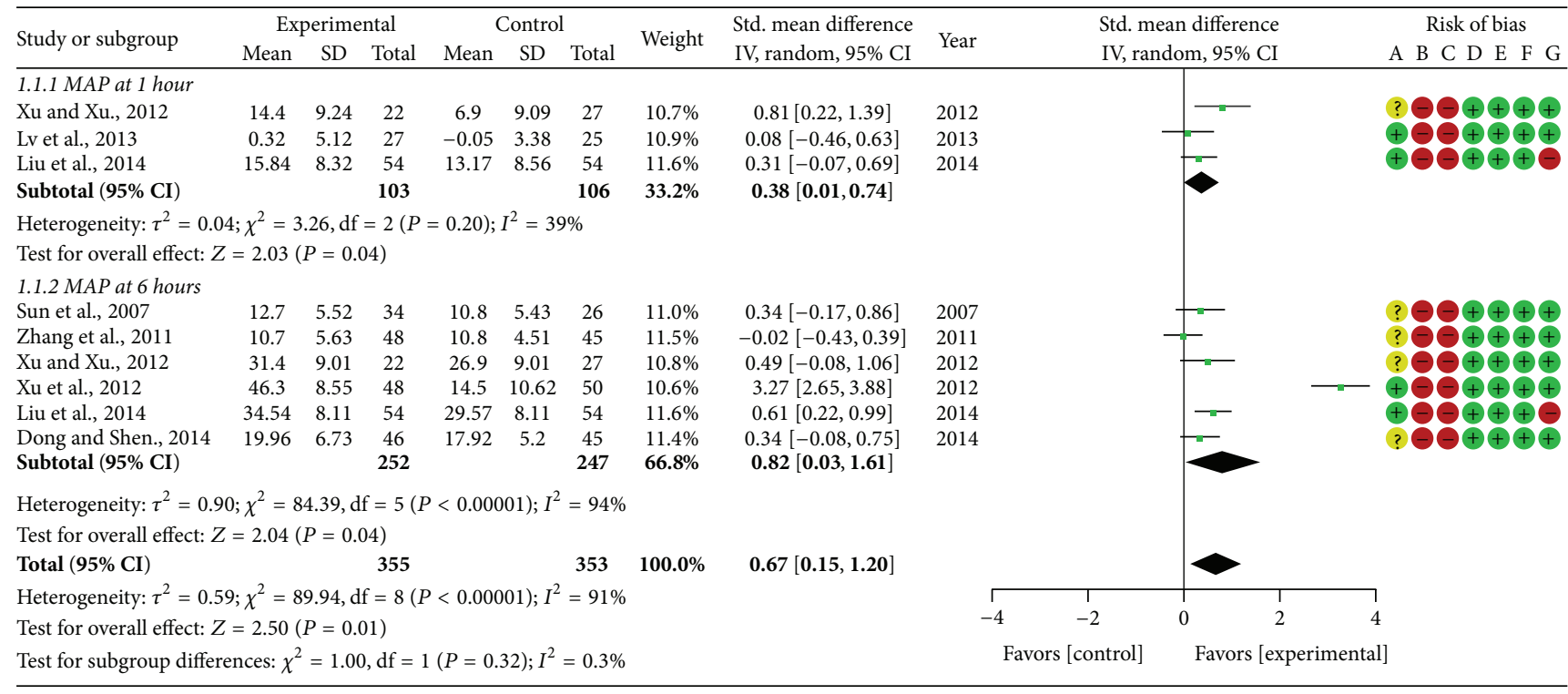

Risk of bias legend
(A) Random sequence generation (selection bias)
(E) Incomplete outcome data (attrition bias)
(B) Allocation concealment (selection bias)
(F) Selective reporting (reporting bias)
(C) Blinding of participants and personnel (performance bias)
(G) Other biases
(D) Blinding of outcome assessment (detection bias)

FIGURE 2: Forest plot of Shenfu injection plus conventional therapy versus conventional therapy: MAP; the authors' judgment about each risk of bias item for each included study.

3.5. Publication Bias. The publication bias was explored via funnel plots (Figures 5, 6, and 7). Points in Figures 5 and 6 presented asymmetry suggesting the possibility of publication bias. Points in Figure 7 seemed to be symmetric, which indicates no obvious publication bias. Given that all these selected articles were published in Chinese academic journals, the potential of publication bias could not be excluded.

\section{Discussion}

In summary, 12 RCTs including 904 patients were selected in our meta-analysis. Based on the findings of the present systematic review, Shenfu injection could further increase MAP, clear serum lactate, normalize HR, and reduce mortality when compared with conventional therapy. However, the potential beneficial effect from Shenfu injection is possibly overstated owing to the generally low methodological qualities of the included RCTs.

The overall methodological quality of our selected trials was judged to be poor, which might reduce the validity and value of the evidence examined in the present review. No studies achieved a low risk of bias as all studies had an unclear or high risk of bias within at least one major domain. Many studies failed to provide experimental methodologies in detail. All the included trials claimed randomization but part of them did not describe information about random sequence generation to estimate whether the randomization procedures had been carried out appropriately. No study employed the strategy of double-blinding, which might lead to the appearance of placebo effect and exaggeration of conclusions. Among the 12 included trials, no study reported number of dropouts and reasons for withdrawal. No study employed 


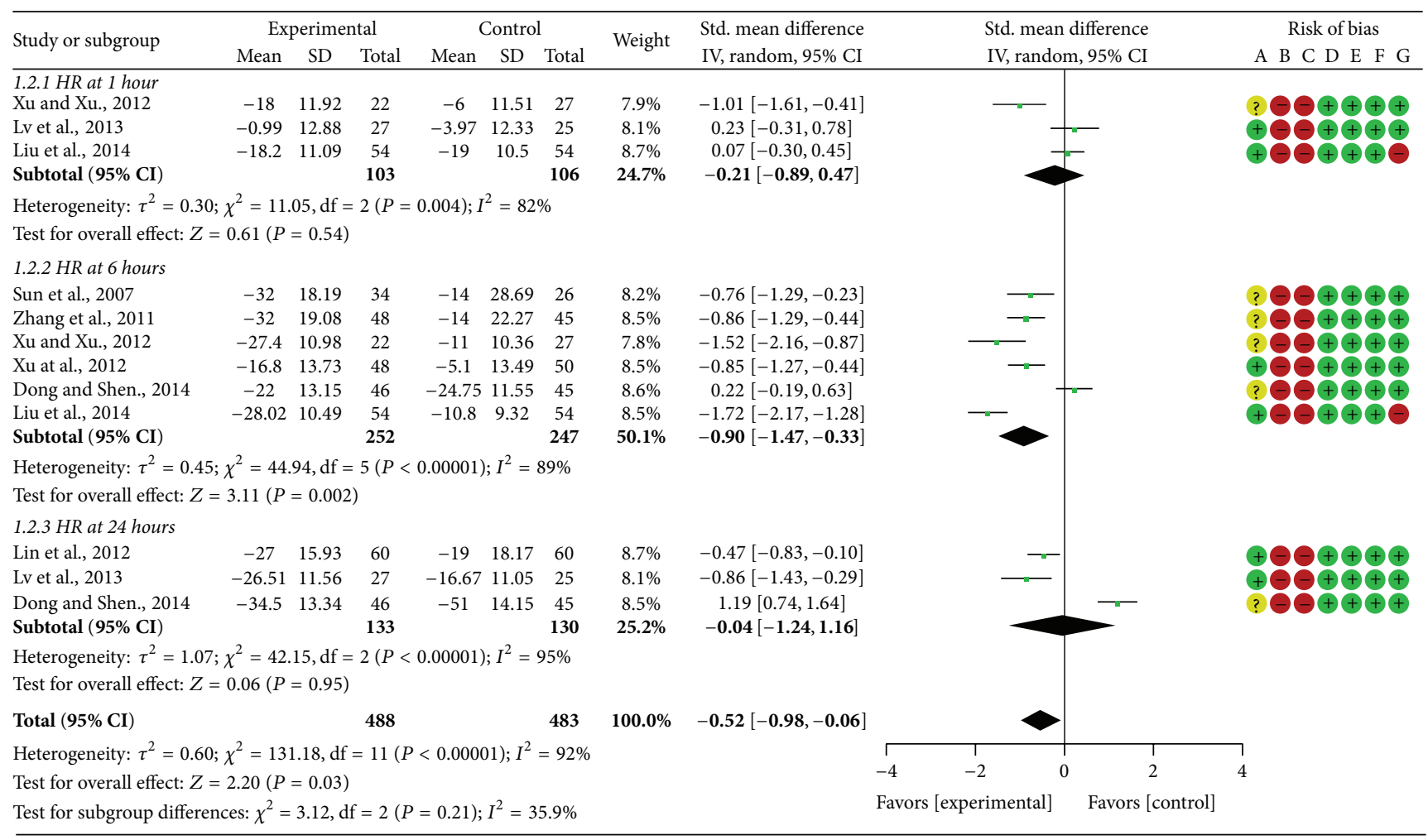

\section{Risk of bias legend}
(A) Random sequence generation (selection bias)
(B) Allocation concealment (selection bias)
(C) Blinding of participants and personnel (performance bias)
(D) Blinding of outcome assessment (detection bias)

(E) Incomplete outcome data (attrition bias)

(F) Selective reporting (reporting bias)

(G) Other biases

FIGURE 3: Forest plot of Shenfu injection plus conventional therapy versus conventional therapy: HR; the authors' judgment about each risk of bias item for each included study.

intention-to-treat analysis, so the conclusions regarding the clinical effect of Shenfu injection might be overstated.

In routine clinical practice, invasive methods such as thermodilution technique are not often indicated, and less invasive techniques, such as echocardiography and pulse contour methods, are not always available [32]. Other hemodynamic variables are frequently used, with satisfactory MAP often a key target, especially in hemodynamically unstable patients [33]. The lactate clearance has also been associated with decreased mortality in patients with severe sepsis and septic shock as well as with multiple organ dysfunction and systemic immunologic activation and inflammation [34]. Lactate clearance should be considered an additional goal of early sepsis resuscitation [35]. Consequently, we included these trials, which employed HR, MAP, serum lactate, and mortality as outcome.

Shenfu injection is an extract of traditional Chinese herbs, which mainly consists of ginsenoside and aconitine [36]. Modern pharmacological research shows that ginsenoside can improve ischemic myocardium metabolism, scavenge free radicals, protect myocardial ultrastructure, and reduce $\mathrm{Ca}^{2+}$ overload, and aconitine can enhance heart contractility, improve coronary circulation, and decrease the effect of acute myocardial ischemia [37]. In addition, aconitine contains noradrenaline salsolinol, which has excitatory effects on $\mathrm{p}$ receptors and $\alpha$-adrenergic receptors, which can significantly increase cerebral blood flow by improving MAP [38]. Shenfu injection could also restore the ability of $\mathrm{Na}^{+}-\mathrm{K}^{+}$-ATPase and $\mathrm{Ca}^{2+}$-ATPase enzyme activities; this may be one of the mechanisms by which Shenfu injection could attenuate the myocardial dysfunction [39]. Moreover, Shenfu injection reduces the expression of TNF- $\alpha$ to block the vicious circle of inflammatory response, improve systemic microcirculation, and prolong the hypoxia tolerance duration [40]. The clinical effect of Shenfu injection was reflected in increase of MAP, reduction of $\mathrm{HR}$, and clearance of serum lactate.

Subgroup analyses were conducted according to the timing of outcome measures. At 1 hour after Shenfu injection treatment, the pooled data revealed no significant difference of HR and serum lactate level between Shenfu group and control group, but the MAP was further increased in Shenfu groups. At 6 hours after treatment, a statistical difference could be detected in all three parameters, suggesting that Shenfu injection started to normalize HR, increase MAP, 


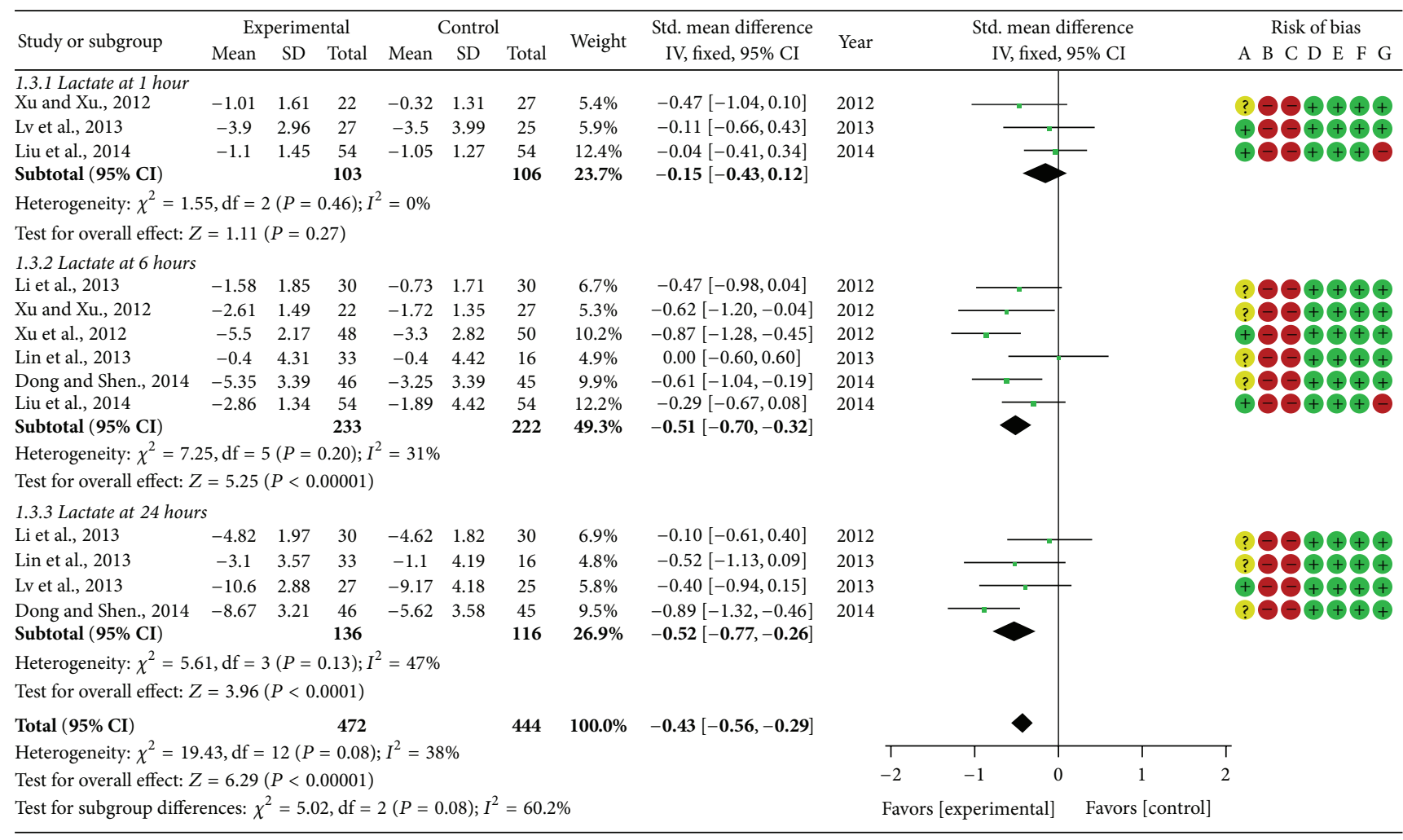

Risk of bias legend
(A) Random sequence generation (selection bias)
(E) Incomplete outcome data (attrition bias)
(B) Allocation concealment (selection bias)
(F) Selective reporting (reporting bias)
(C) Blinding of participants and personnel (performance bias)
(G) Other biases
(D) Blinding of outcome assessment (detection bias)

Figure 4: Forest plot of Shenfu injection plus conventional therapy versus conventional therapy: lactate; the authors' judgment about each risk of bias item for each included study.

and help to clear serum lactate within 6 hours. And even at 24 hours after treatment, lactate level was further reduced in Shenfu injection group compared to control group. In terms of HR, there was no significant difference between Shenfu injection and conventional therapy at 24 hours after treatment. The findings of our work indicate that Shenfu injection helps to improve organ perfusion by increasing MAP, cut down the load of the heart, and reduce the oxygen consumption in early recovery of septic shock. In addition, the reduced blood lactate concentration suggests that Shenfu injection is capable of improving tissue hypoperfusion and the microcirculation. In a word, Shenfu injection was effective in the treatment of septic shock.

Limitations. In our present review, we included these studies which employed HR, MAP, serum lactate, and mortality as outcome. Changes of these parameters reflected the improvement of microcirculation and protective effect on myocardial cells of Shenfu injection. However, these adopted indicators are not comprehensive enough for the systematic assessment of Shenfu injection. Some well-established indicators such as APACHE/SAPS/SOFA scores, mechanical ventilation rates, renal replacement, or acute kidney injury rates were not reported by our selected trials.

Results of our systematic review were somewhat limited due to the poor methodological quality of included trials. Future randomized controlled trials should employ improved methodologies and reporting specifications as follows: (a) all clinical studies of Shenfu injection should be registered and comply with the Consolidated Standards of Reporting Trials (CONSORT) statement; (b) the sample sizes should be calculated before the start of the trials; (c) the generation of random allocation sequences and allocation concealment should be provided in detail; (d) these studies should be double-blinded and placebo-controlled; (e) the standard of diagnosis should be unified and widely accepted; (f) all adverse events associated with Shenfu injection should be reported and rigorously assessed.

\section{Conclusion}

Results of the present systematic review suggest that Shenfu injection could further increase MAP, normalize HR, clear blood lactate, and reduce mortality when compared with 


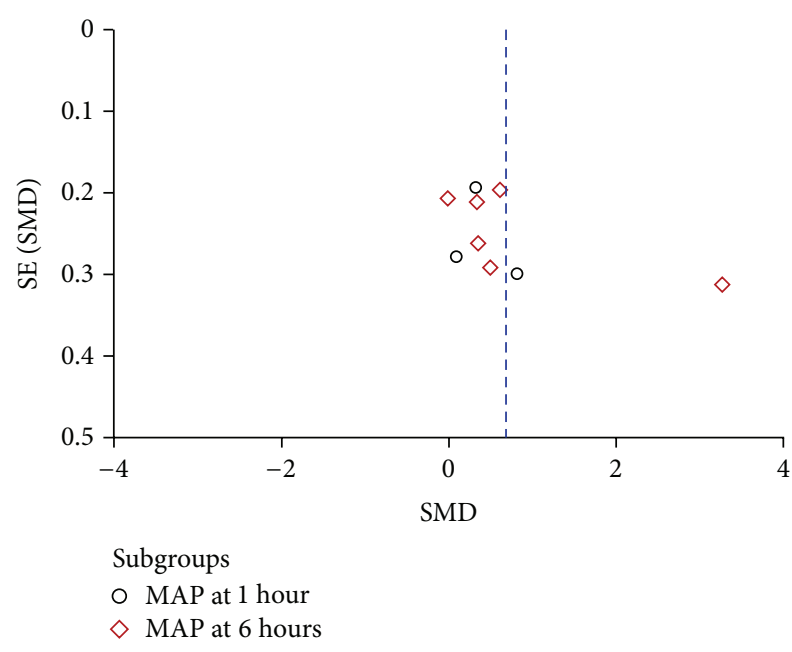

Figure 5: Funnel plot of Shenfu injection plus conventional therapy versus conventional therapy: MAP.

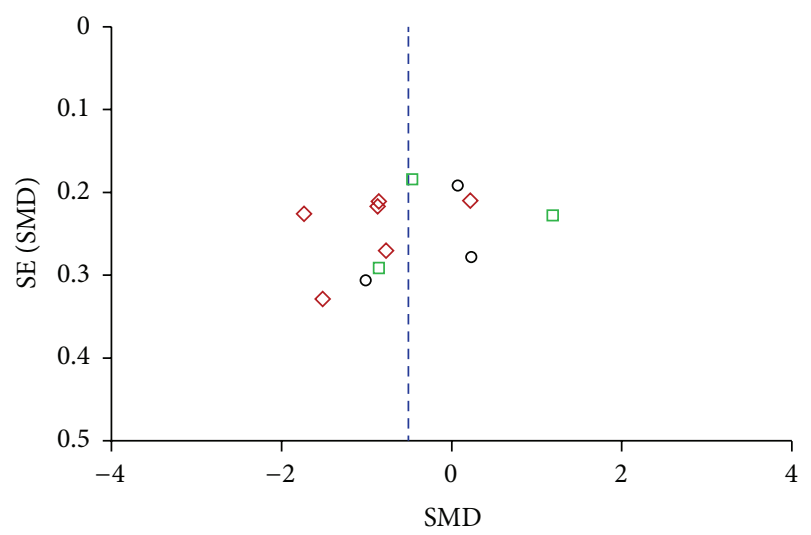

Subgroups
$\bigcirc$ HR at 1 hour $\quad \square$ HR at 24 hours
$\diamond$ HR at 6 hours

FIGURE 6: Funnel plot of Shenfu injection plus conventional therapy versus conventional therapy: HR.

conventional therapy. However, the conclusion should be interpreted cautiously due to the poor methodological qualities of included studies. Additional RCTs with large-scale, rigorous study design and strict reporting specification are required.

\section{Conflict of Interests}

The authors declare that there is no conflict of interests regarding the publication of this paper.

\section{Authors' Contribution}

Yi Li and Xuezhong Yu produced the idea to do the analyses and made the final version of the paper. Zhengtao Lv and Zijun Mou did the reference search and evaluated the data,

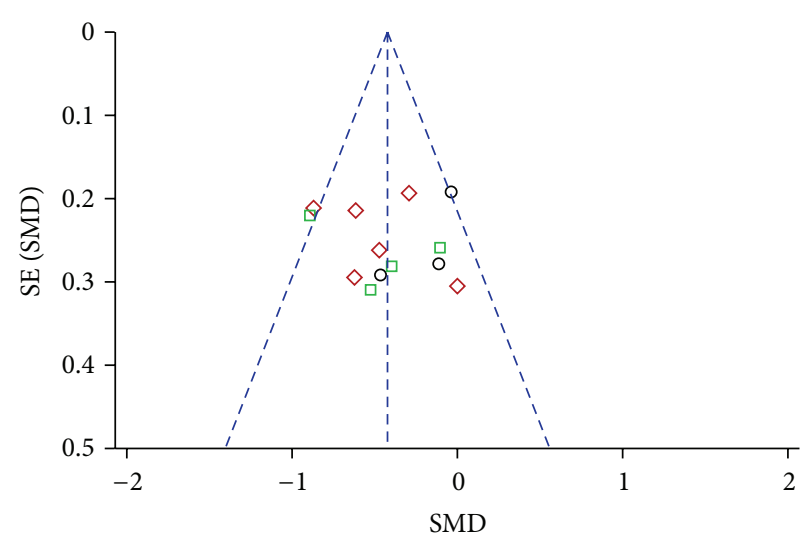

Subgroups

$\bigcirc$ Lactate at 1 hour $\quad \square$ Lactate at 24 hours
$\diamond$ Lactate at 6 hours

FIGURE 7: Funnel plot of Shenfu injection plus conventional therapy versus conventional therapy: lactate.

and both contributed equally to this paper. Zhengtao Lv and Zijun Mou contributed equally to this work. All the authors contributed to the work and development of this paper.

\section{Acknowledgment}

This study was supported by the National Key Medical Department Grant of China (Grant no. 2012-650).

\section{References}

[1] P. Asfar, F. Meziani, J.-F. Hamel et al., "High versus low bloodpressure target in patients with septic shock," The New England Journal of Medicine, vol. 370, no. 17, pp. 1583-1593, 2014.

[2] A. Thooft, R. Favory, D. R. Salgado et al., "Effects of changes in arterial pressure on organ perfusion during septic shock," Critical Care, vol. 15, no. 5, article R222, 2011.

[3] J.-L. Vincent, Y. Sakr, C. L. Sprung et al., "Sepsis in European intensive care units: results of the SOAP study," Critical Care Medicine, vol. 34, no. 2, pp. 344-353, 2006.

[4] The ARISE Investigators and the ANZICS Clinical Trials Group, "Goal-directed resuscitation for patients with early septic shock," The New England Journal of Medicine, vol. 371, no. 16, pp. 1496-1506, 2014.

[5] C. Pierrakos, D. Velissaris, S. Scolletta, S. Heenen, D. de Backer, and J.-L. Vincent, "Can changes in arterial pressure be used to detect changes in cardiac index during fluid challenge in patients with septic shock?" Intensive Care Medicine, vol. 38, no. 3, pp. 422-428, 2012.

[6] J. A. Russell, "Is there a good MAP for septic shock?" The New England Journal of Medicine, vol. 370, no. 17, pp. 1649-1651, 2014.

[7] R. P. Dellinger, M. M. Levy, J. M. Carlet et al., "Surviving Sepsis Campaign: international guidelines for management of severe sepsis and septic shock: 2008," Critical Care Medicine, vol. 36, no. 1, pp. 296-327, 2008.

[8] M. Varpula, M. Tallgren, K. Saukkonen, L.-M. Voipio-Pulkki, and V. Pettilä, "Hemodynamic variables related to outcome in septic shock," Intensive Care Medicine, vol. 31, no. 8, pp. 10661071, 2005. 
[9] A. Morelli, C. Ertmer, M. Westphal et al., "Effect of heart rate control with esmolol on hemodynamic and clinical outcomes in patients with septic shock: a randomized clinical trial," The Journal of the American Medical Association, vol. 310, no. 16, pp. 1683-1691, 2013.

[10] O. Sander, I. D. Welters, P. Foëx, and J. W. Sear, "Impact of prolonged elevated heart rate on incidence of major cardiac events in critically ill patients with a high risk of cardiac complications," Critical Care Medicine, vol. 33, no. 1, pp. 81-88, 2005.

[11] C. A. Schmittinger, C. Torgersen, G. Luckner, D. C. H. Schröder, I. Lorenz, and M. W. Dünser, "Adverse cardiac events during catecholamine vasopressor therapy: a prospective observational study," Intensive Care Medicine, vol. 38, no. 6, pp. 950-958, 2012.

[12] A. Morelli, A. Donati, C. Ertmer et al., "Microvascular effects of heart rate control with esmolol in patients with septic shock: a pilot study," Critical Care Medicine, vol. 41, no. 9, pp. 2162-2168, 2013.

[13] A. Rudiger and M. Singer, "The heart in sepsis: From basic mechanisms to clinical management," Current Vascular Pharmacology, vol. 11, no. 2, pp. 187-195, 2013.

[14] P. Wacharasint, T.-A. Nakada, J. H. Boyd, J. A. Russell, and K. R. Walley, "Normal-range blood lactate concentration in septic shock is prognostic and predictive," Shock, vol. 38, no. 1, pp. 410, 2012.

[15] D. O. Thomas-Rueddel, B. Poidinger, M. Weiss et al., "Hyperlactatemia is an independent predictor of mortality and denotes distinct subtypes of severe sepsis and septic shock," Journal of Critical Care, vol. 30, no. 2, pp. 439.e1-439.e6, 2015.

[16] P. J. D. Andrews, "Early goal-directed therapy in the emergency department-lactate is biomarker of illness severity, but of which disease process?” Resuscitation, vol. 82, no. 10, pp. 12631264, 2011.

[17] M. A. Puskarich, S. Trzeciak, N. I. Shapiro et al., "Whole blood lactate kinetics in patients undergoing quantitative resuscitation for severe sepsis and septic shock," Chest, vol. 143, no. 6, pp. 1548-1553, 2013.

[18] G. Dong and B. Shen, "Clinical effect of Shenfu injection in the sepsis bundle treatment of septic shock," Journal of Emergency in Traditional Chinese Medicine, vol. 23, no. 5, pp. 944-945, 2014.

[19] Y. He, Q. Wang, and X. Wu, "Effect of early used high dose Shenfu injection for the treatment of septic shock," Journal of Emergency in Traditional Chinese Medicine, vol. 22, no. 1, pp. 99-100, 2013.

[20] J. P. T. Higgins and S. Green, Eds., Cochrane Handbook for Systematic Reviews of Interventions, Version 5.1.0, The Cochrane Collaboration, 2011, http://www.cochrane-handbook.org/.

[21] R. P. Dellinger, J. M. Carlet, H. Masur et al., "Surviving Sepsis Campaign guidelines for management of severe sepsis and septic shock," Intensive Care Medicine, vol. 30, no. 4, pp. 536$555,2004$.

[22] W. Liu, Q. Liang, T. Jin, D. Luo, and G. Chen, “Clinical effect of Shenfu injection combined with norepinephrine in treatment of septic shock," Journal of Emergency in Traditional Chinese Medicine, vol. 23, no. 8, pp. 1550-1552, 2014.

[23] G. Lv, D. Zhuo, S. Xiong, L. Min, and S. Qiao, "Effect of Shenfu injection on oxygen metabolism in patients with septic shock," Journal of Jiangxi University of Traditional Chinese Medicine, vol. 25, no. 1, pp. 30-32, 2013.

[24] S. Sun, Y. Liu, and R. Wang, "Study of Shenfu injection on serius sepsis and septic shock recovery of the early intervention,"
Journal of Emergency in Traditional Chinese Medicine, vol. 16, no. 10, pp. 1214-1215, 2007.

[25] L. Xu and L. Xu, "Shenfu injection combined with dopamine in the early targeted treatment of septic shock," Journal of Emergency in Traditional Chinese Medicine, vol. 21, no. 11, pp. 1740-1741, 2012.

[26] Y. Xu, B. Chen, L. Zhong, and H. Wu, "Study of integrated therapy in the early recovery of septic shock," Soft Science of Health, vol. 26, no. 6, pp. 578-580, 2013.

[27] W. Zhang, B. Wu, J. Guan et al., "Research on septic shock treated with injection of Shenfu," Medical Information, no. 9, pp. 4903-4904, 2011.

[28] M. Lin, S. Wang, and Y. Dong, "Clinical observation of Shenfu injection in the treatment of septic shock," Journal of Emergency in Traditional Chinese Medicine, vol. 21, no. 7, pp. 1132-1133, 2012.

[29] P. Li, B. Liu, and J. Fu, "Effect of Shenfu injection combined with ulinastatin on blood lactate in patients with septic shock," Shaanxi Journal of Traditional Chinese Medicine, vol. 33, no. 10, p. 1337, 2013.

[30] H. Lin, C. Ma, R. Wang, and D. Liang, "Efect of Shenfu injection on blood lactate and prognosis in patients with severe pneumonia," Journal of Emergency in Traditional Chinese Medicine, vol. 22, no. 2, pp. 199-200, 2013.

[31] S. Sun, X. Mo, and C. Ma, "Shenfu injection early intervention on the organ function with septic shock," Journal of Emergency in Traditional Chinese Medicine, vol. 17, no. 10, pp. 1392-1393, 2008.

[32] J.-L. Vincent, M. R. Pinsky, C. L. Sprung et al., "The pulmonary artery catheter: in medio virtus," Critical Care Medicine, vol. 36, no. 11, pp. 3093-3096, 2008.

[33] P. E. Marik and J. Varon, "The hemodynamic derangements in sepsis: Implications for treatment strategies," Chest, vol. 114, no. 3, pp. 854-860, 1998.

[34] A. M. Napoli and T. A. Seigel, "The role of lactate clearance in the resuscitation bundle," Critical Care, vol. 15, no. 5, article 199, 2011.

[35] M. A. Puskarich, "Emergency management of severe sepsis and septic shock," Current Opinion in Critical Care, vol. 18, no. 4, pp. 295-300, 2012.

[36] L. Huang and J. Cao, "The protective effects of Shen-Fu injection on experimental acute pancreatitis in a rat model," Oxidative Medicine and Cellular Longevity, vol. 2014, Article ID 248786, 7 pages, 2014.

[37] W.-T. Song, F.-F. Cheng, L. Xu, C.-R. Lin, and J.-X. Liu, "Chinese medicine shenfu injection for heart failure: a systematic review and meta-analysis," Evidence-based Complementary and Alternative Medicine, vol. 2012, Article ID 713149, 25 pages, 2012.

[38] W. Gu, X. Hou, H. Zhou, and C. Li, "Protective effect of shen-fu injection on neuronal mitochondrial function in a porcine model of prolonged cardiac arrest," Evidence-Based Complementary and Alternative Medicine, vol. 2014, Article ID 523847, 8 pages, 2014.

[39] Q. Zhang and C. Li, "The roles of traditional chinese medicine: Shen-Fu injection on the postresuscitation care bundle," Evidence-Based Complementary and Alternative Medicine, vol. 2013, Article ID 319092, 7 pages, 2013.

[40] Q. Zhang, C.-S. Li, S. Wang, and W. Gu, "Effects of Chinese medicine Shen-Fu injection on the expression of inflammatory cytokines and complements during post-resuscitation immune dysfunction in a porcine model," Chinese Journal of Integrative Medicine, 2014. 


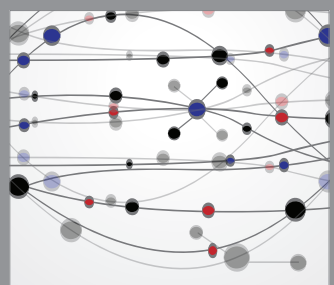

The Scientific World Journal
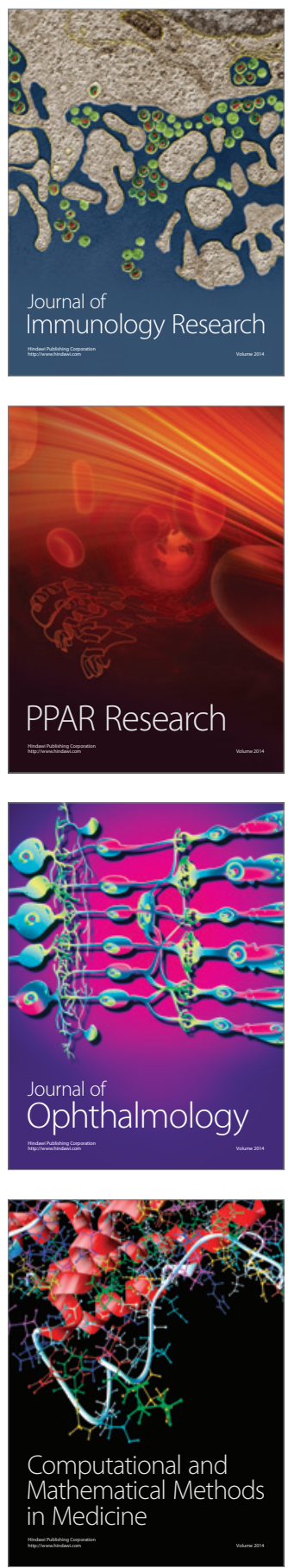

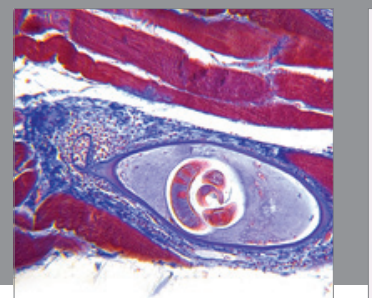

Gastroenterology

Research and Practice
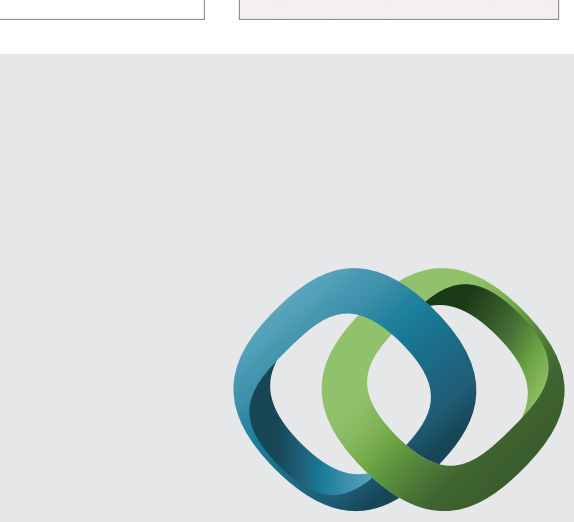

\section{Hindawi}

Submit your manuscripts at

http://www.hindawi.com
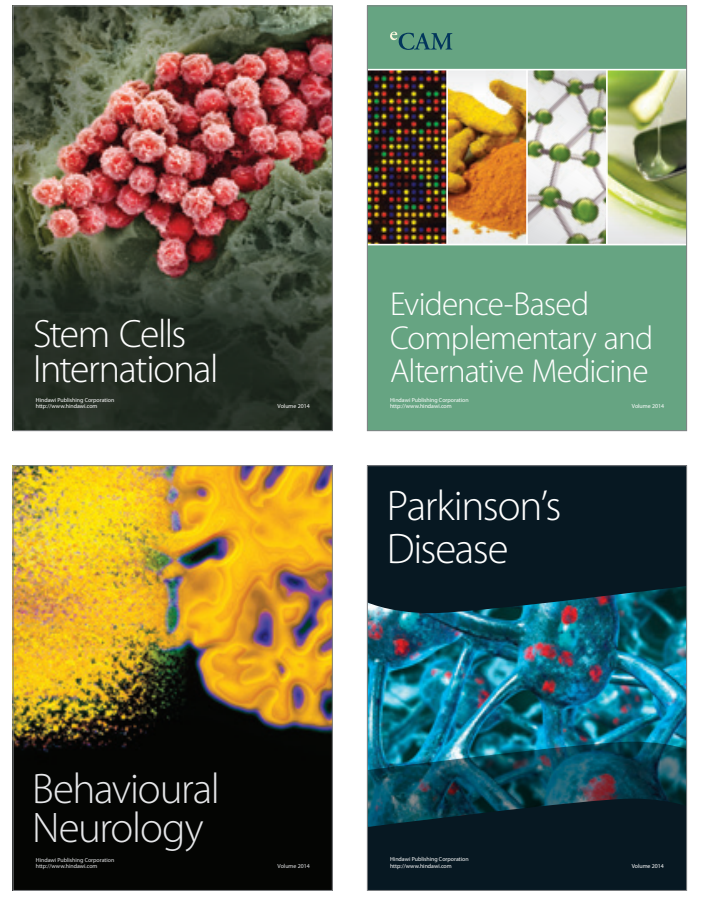
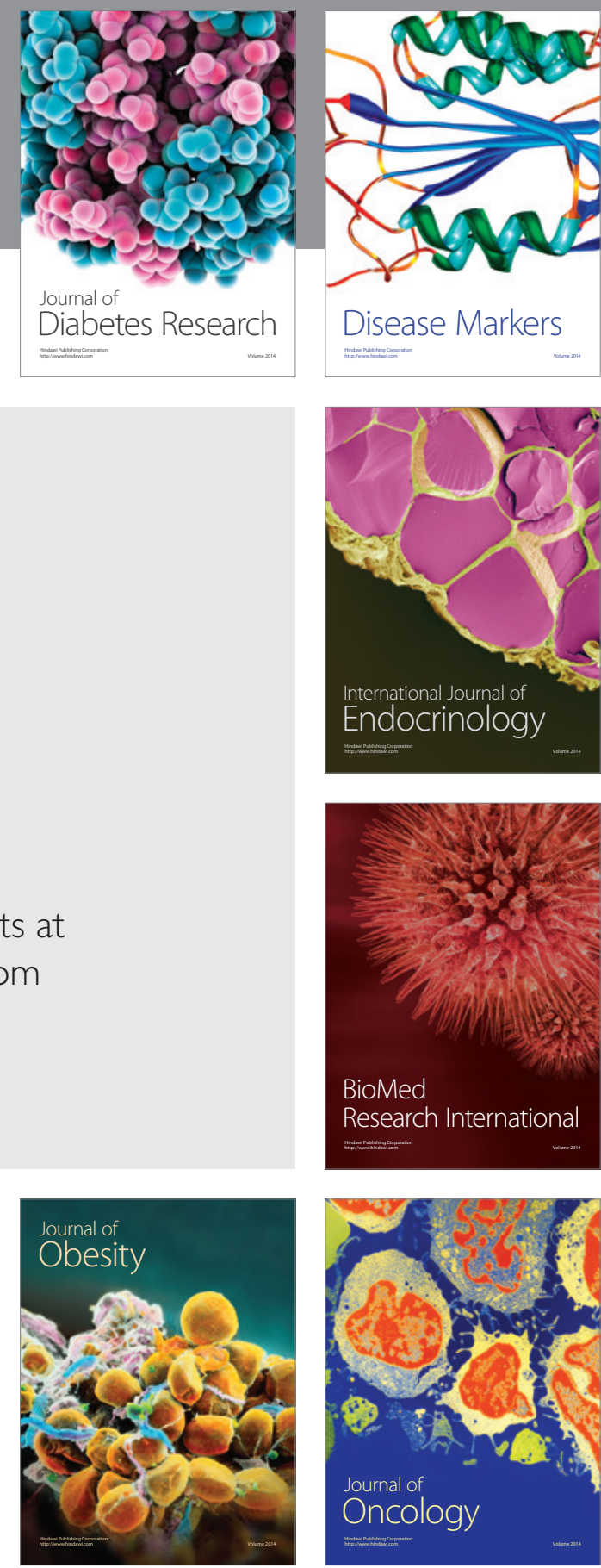

Disease Markers
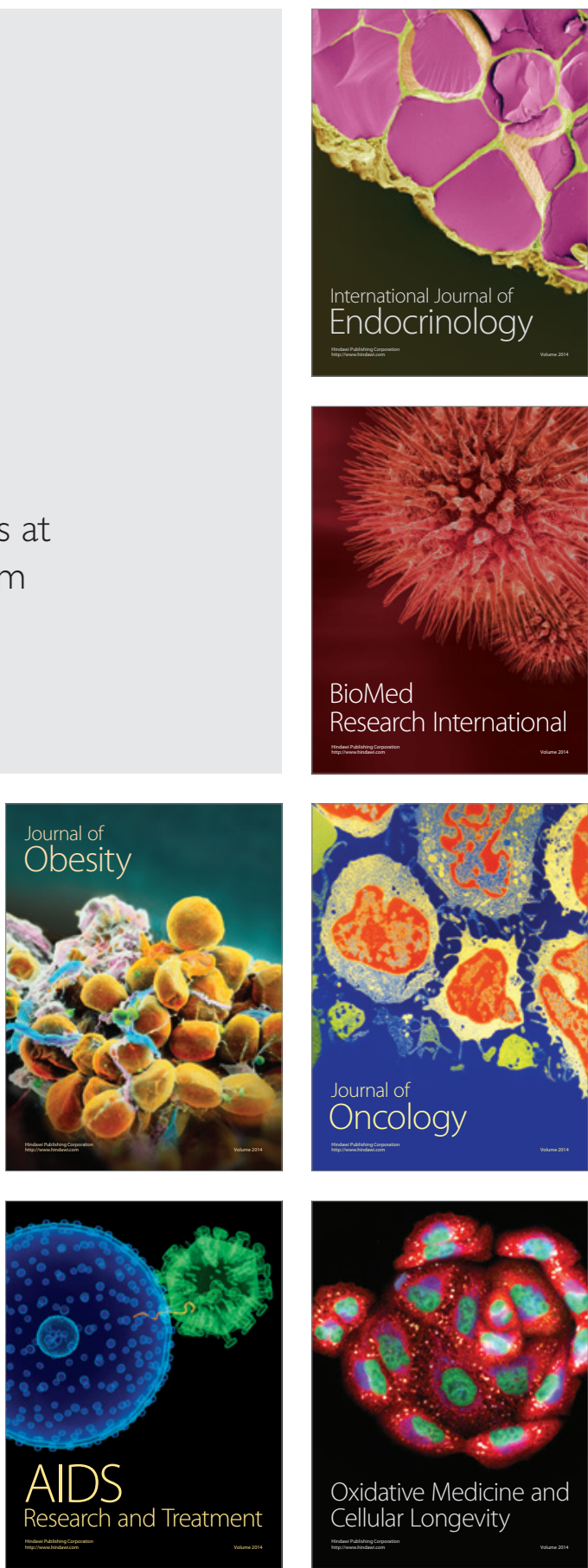